\title{
Metagonimus yokogawai
}

National Cancer Institute

\section{Source}

National Cancer Institute. Metagonimus yokogawai. NCI Thesaurus. Code C123514.

A species of parasitic flatworms in the family Heterophyidae. The lifecycle of M.

yokog awai involves snails as the first intermediate host, freshwater fish as the second

intermediate host, and humans, fish-eating carnivores, or birds as the definitive host.

Infestation causes metagonimiasis. 